\title{
CARGAS DE TRABALHO E A SAÚDE DO TRABALHADOR DE ENFERMAGEM: REVISÃO INTEGRATIVA*
}

\author{
Deciane Pintanela de Carvalho ${ }^{1}$, Laurelize Pereira Rocha ${ }^{2}$, Jamila Geri Tomaschewski Barlem², Jennifer Specht \\ Dias $^{1}$, Claudia Denise Schallenberger ${ }^{1}$
}

RESUMO: Este estudo tem como objetivo identificar a influência das cargas de trabalho na saúde do trabalhador de enfermagem. A busca foi realizada em novembro de 2016, em quatro bases de dados, estabelecendo-se critérios de inclusão e exclusão, sendo selecionados 50 estudos. Os resultados destacam os conceitos, tipos e condições de trabalho que predispõem as cargas de trabalho, evidenciando as consequências na saúde da equipe de enfermagem por meio dos adoecimentos, que acarretam afastamentos e absenteísmo, estresse, desgastes físicos e emocionais, riscos para acidentes de trabalho, entre outros. Conclui-se que a identificação dessas influências possibilita a implementação de ações organizacionais, de prevenção de acidentes e doenças relacionadas ao trabalho.

DESCRITORES: Carga de trabalho; Saúde do trabalhador; Absenteísmo; Enfermagem.

\section{WORKLOADS AND NURSING WORKERS' HEALTH: INTEGRATIVE REVIEW}

\begin{abstract}
This study aimed to identify the effects of workloads on the health status of nursing workers. The search was conducted in November 2016, at four databases, and inclusion and exclusion criteria were established. At the end, 50 studies were selected. The results highlight work concepts, types and conditions, demonstrating their effects on the health status of the nursing team, which is manifested through illnesses that result in temporary absence from work (sick leaves) and absenteeism, stress, physical and emotional fatigue, risks of occupational accidents, among others. It is concluded that the identification of these effects allows the implementation of organizational actions targeted to the prevention of occupational accidents and diseases.
\end{abstract}

DESCRIPTORS: Workload; Worker's health; Absenteeism; Nursing.

\section{CARGAS DE TRABAJO Y LA SALUD DEL TRABAJADOR DE ENFERMERÍA: REVISIÓN INTEGRATIVA}

RESUMEN: Estudio cuyo objetivo fue identificar la influencia de las cargas de trabajo en la salud del trabajador de enfermería. La búsqueda fue realizada en noviembre de 2016, en cuatro bases de datos, bajo los criterios de inclusión y exclusión, siendo seleccionados 50 estudios. Se destacan los conceptos, tipos y condiciones de trabajo que predisponen las cargas de trabajo, evidenciando las consecuencias en la salud del equipo de enfermería por medio de las enfermedades, las cuales resultan alejamiento y absentismo, estrés, desgastes físicos y emocionales, riesgos para accidentes de trabajo, entre otros. Se constata que la identificación de esas influencias posibilita la implementación de acciones organizacionales, de prevención de accidentes y enfermedades referentes al trabajo.

DESCRIPTORES: Carga de trabajo; Salud del trabajador; Absentismo; Enfermería.

\footnotetext{
*Artigo extraído da dissertação intitulada: “Cargas de trabalho e perda de produtividade dos trabalhadores de enfermagem:
} estudo em um Hospital Universitário". Universidade Federal do Rio Grande, 2016.

1Enfermeira. Mestranda em Enfermagem. Universidade Federal do Rio Grande. Rio Grande, RS, Brasil.

${ }^{2}$ Enfermeira. Doutora em Enfermagem. Docente de Enfermagem da Universidade Federal do Rio Grande. Rio Grande, RS, Brasil.

\section{Autor Correspondente:}

Deciane Pintanela de Carvalho

Universidade Federal do Rio Grande

R. General Osório, s/n - 96200-400 - Rio Grande, RS, Brasil

E-mail: deciane.carvalho@gmail.com
Recebido: 02/05/2016

Finalizado: 17/12/2016 


\section{- INTRODUÇÃO}

A equipe de enfermagem representa a maior força de trabalho presente nas instituições hospitalares, entretanto durante a realização da assistência existem limitações quanto ao número de trabalhadores e recursos materiais disponíveis, o que torna o trabalho desgastante, em vistas das cargas de trabalho presentes no ambiente de trabalho(1). As cargas de trabalho são inerentes ao próprio processo e ambiente de trabalho, exercendo influência direta e indiretamente na saúde do trabalhador, sendo necessária sua identificação e compreensão durante o processo laboral ${ }^{(2)}$.

Os trabalhadores de enfermagem estão expostos a distintas cargas de trabalho, as quais podem ser classificadas em biológicas, como, por exemplo, os fluídos corpóreos; químicas, por meio da utilização de medicamentos; mecânicas, identificadas pelos acidentes com agulhas e materiais perfurocortantes; fisiológicas, pode-se destacar o trabalho em pé e postura inadequada; e psíquicas, ligadas às condições inadequadas de trabalho, sendo estes alguns dos fatores determinantes das cargas de trabalho, as quais estão relacionadas ao processo de adoecimento do trabalhador ${ }^{(3)}$.

A exposição contínua às cargas de trabalho, a vivência diária de sentimentos de prazer e de sofrimento gera desgaste no trabalhador, e isto pode evoluir para o adoecimento. Desta forma, é necessário que o trabalhador compreenda as peculiaridades da profissão, e aproprie-se de estratégias que possam reduzir este desgaste profissional, buscando a preservação da sua saúde ${ }^{(4)}$.

Os desgastes advindos desta exposição são responsáveis pelos acidentes de trabalho e afastamentos por meio de licenças médicas, que podem estar relacionados às dores osteoarticulares, torções, fraturas, luxações, contusões e incapacidade motora, alterações de humor, ansiedade, depressão, estresse, insônia e doenças infectocontagiosas ${ }^{(5)}$.

Neste sentido, o estudo das cargas de trabalho permite identificar os problemas decorrentes do processo de trabalho que influenciam a saúde do trabalhador e, desta forma, promover ações de promoção de saúde e prevenção de doenças entre trabalhadores de enfermagem. Assim, este estudo tem como objetivo identificar a influência das cargas de trabalho na saúde do trabalhador de enfermagem.

\section{MÉTODO}

Trata-se de uma revisão integrativa realizada por meio de seis etapas: 1) definição da questão norteadora; 2) estabelecimento de critérios de inclusão/exclusão; 3) definição das informações a serem extraídas dos artigos; 4) análise dos estudos incluídos; 5) interpretação dos resultados; e 6) apresentação da revisão integrativa ${ }^{(6)}$.

Como questão norteadora utilizou-se: Qual a influência das cargas de trabalho na saúde do trabalhador de enfermagem? A coleta dos artigos foi realizada nas bases de dados Medical Literature Analysis and Retrieval System Online (MEDLINE), Literatura Latino-Americana e do Caribe em Ciências da Saúde (LILACS), Scientific Electronic Library Online (SciELO) e Cumulative Index to Nursing and Allied Health Literature (CINAHL) em novembro de 2016.

Para a seleção dos artigos, utilizou-se os descritores controlados conforme a classificação dos Descritores em Ciências da Saúde (DeCS): Enfermagem (Nursing), Carga de trabalho (Workload) e Absenteísmo (Absenteeism). A busca foi refinada utilizando-se o operador booleano AND, o qual permitiu realizar as seguintes combinações: Carga de trabalho x Enfermagem; Workload x Nursing; Absenteísmo x Enfermagem; Absenteeism x Nursing. Este cruzamento foi realizado com a finalidade de verificar se existem estudos científicos que comprovem a relação entre carga de trabalho e absenteísmo. A Tabela 1 apresenta os resultados da busca por descritores nas bases de dados.

Foram instituídos como critérios de inclusão: artigos científicos, em português, inglês ou espanhol, disponibilidade na íntegra, gratuitamente e publicados no período de 2010 a 2016. Como critérios de exclusão optou-se em: excluir teses e dissertações, textos sem disponibilidade de resumo para primeira apreciação, e os artigos repetidos foram considerados uma única vez. 
Tabela 1 - Resultados dos cruzamentos dos descritores nas bases de dados. Rio Grande, RS, Brasil, 2016

\begin{tabular}{lcc} 
Bases de dados & \multicolumn{2}{c}{ Descritores } \\
\cline { 2 - 3 } & Carga de trabalho $x$ enfermagem & Absenteísmo $\mathbf{x}$ Enfermagem \\
\cline { 2 - 3 } & Workload $\boldsymbol{x}$ Nursing & Absenteeism $\mathbf{x}$ Nursing \\
\hline MEDLINE & 168 & 41 \\
\hline LILACS & 34 & 22 \\
\hline SciELO & 351 & 135 \\
\hline CINAHL & 5.152 & 738 \\
\hline Total & 5.705 & 936 \\
\hline
\end{tabular}

A coleta totalizou 6.641 artigos, após aplicou-se os critérios de inclusão e exclusão, realizou-se leitura dos resumos e leitura dos artigos na íntegra para seleção da amostra. Desta forma, fizeram parte da amostra 50 artigos, conforme é apresentado na Figura 1.

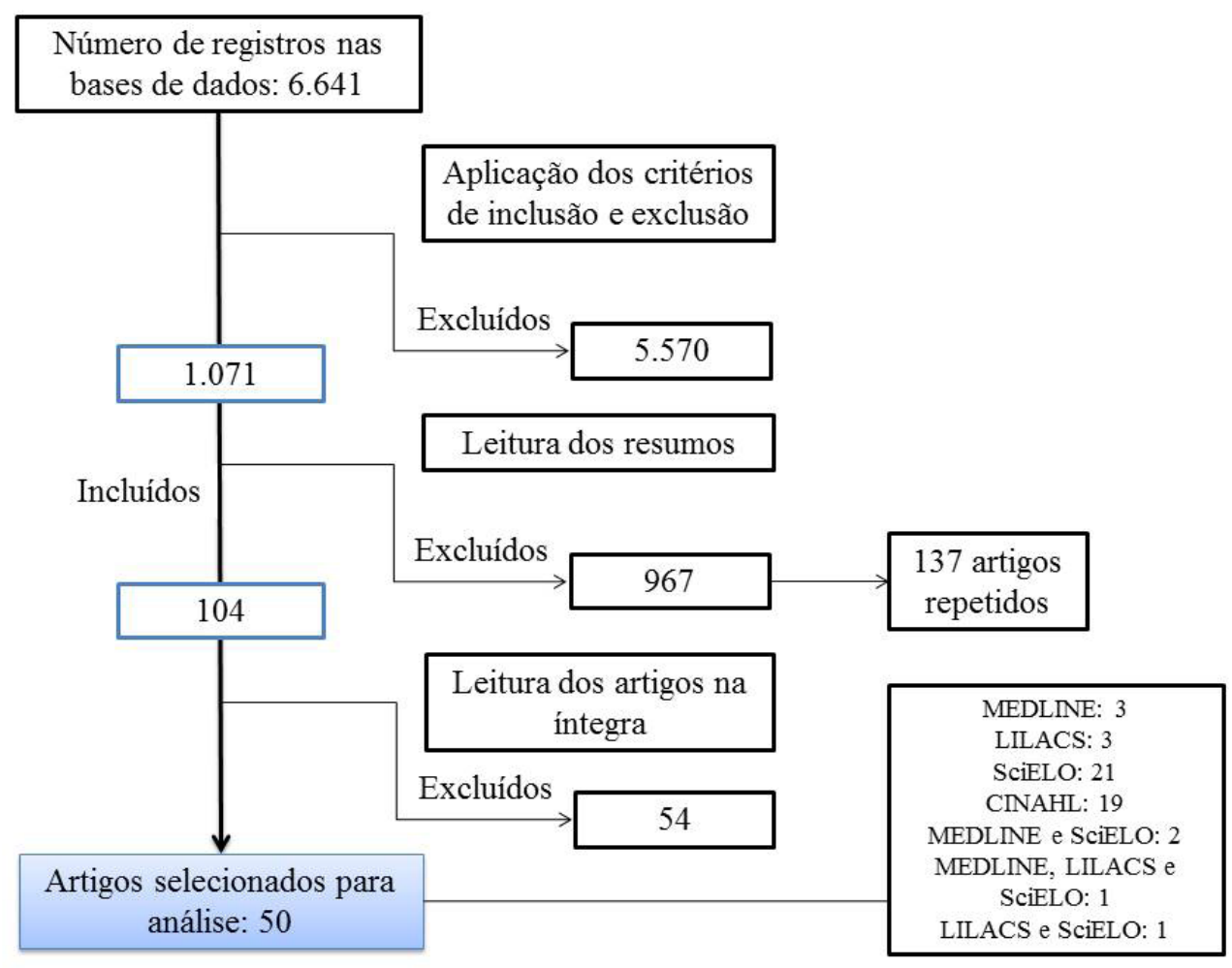

Figura 1 - Diagrama da seleção da amostra. Rio Grande, RS, Brasil, 2016

Posteriormente, prosseguiu-se a análise das publicações quanto à identificação do título do artigo, base de dados em que foi extraído, periódico, ano de publicação, país de origem, tipo de estudo e o nível de evidência (NE).

O nível de evidência caracteriza a maneira como as evidências são classificadas segundo forma hierárquica e de acordo com a abordagem metodológica adotada. Desta forma, as evidências são resultantes de: nível 1 (revisão sistemática ou metanálise de múltiplos estudos clínicos randomizados); nível 2 (ensaios clínicos randomizados bem desenhados); nível 3 (ensaios clínicos bem desenhados sem randomização); nível 4 (estudos de caso-controle e de coorte); nível 5 (revisões sistemáticas de estudos descritivos e qualitativos); nível 6 (estudos descritivos ou de abordagem qualitativa); nível 7 (opinião de especialistas) $)^{(7)}$. 
Dos 50 artigos analisados, identificou-se que o maior número de publicações ocorreu no ano de 2013, com 13 artigos; seguido de 2011 com 10 artigos, 2012 e 2015 com nove artigos cada. De acordo com o país de origem dos artigos, 20 estudos foram desenvolvidos no Brasil, nos países Colômbia, Espanha e Estados Unidos da América foram publicados três artigos cada, e no Chile, África e Austrália dois artigos cada.

Com relação aos periódicos, 23 estudos foram publicados em periódicos nacionais e 27 em periódicos internacionais. O tipo de estudo mais utilizado nas metodologias foram os estudos quantitativos, em 29 artigos; e do tipo qualitativo, em 10 artigos. O nível de evidência que mais caracterizou a amostra foi o nível 6 com 43 artigos. No Quadro 1 apresenta-se uma síntese dos estudos analisados nesta revisão.

Quadro 1 - Caracterização dos estudos segundo periódico, ano e país de origem, tipo de estudo e nível de evidência (NE). Rio Grande, RS, Brasil, 2016 (continua)

\begin{tabular}{|c|c|c|c|c|}
\hline Periódico & Ano & País & Tipo de estudo & NE \\
\hline Rev. Latino-Am. Enfermagem ${ }^{(8)}$ & 2013 & Brasil & Quantitativo & 6 \\
\hline Int. Arch. Occup. Environ. Health. ${ }^{(9)}$ & 2013 & Polônia & Quantitativo & 6 \\
\hline Ind. Health. ${ }^{(10)}$ & 2013 & Taiwan & Quantitativo & 6 \\
\hline Rev Gaúcha Enferm. ${ }^{(11)}$ & 2011 & Brasil & Rev. Integrativa & 5 \\
\hline Av. enferm. ${ }^{(12)}$ & 2010 & Colômbia & Qualitativo & 6 \\
\hline Rev. Portuguesa de Enf. de Saúde Mental ${ }^{(13)}$ & 2014 & Portugal & Quantitativo & 6 \\
\hline Ciênc. saúde coletiva. ${ }^{(14)}$ & 2011 & Brasil & Qualitativo & 6 \\
\hline Ciênc. saúde coletiva.(15) & 2011 & Brasil & Qualitativo & 6 \\
\hline Rev. Latino-Am. Enfermagem ${ }^{(16)}$ & 2011 & Brasil & Qualitativo & 6 \\
\hline Rev. Latino-Am. Enfermagem ${ }^{(17)}$ & 2014 & Brasil & Quantitativo & 6 \\
\hline Rev. bras. epidemiol. ${ }^{(18)}$ & 2012 & Brasil & Quantitativo & 6 \\
\hline Av. enferm. ${ }^{(19)}$ & 2013 & Colômbia & Rev. Bibliográfica & 5 \\
\hline Rev. Latino-Am. Enfermagem ${ }^{(20)}$ & 2010 & Brasil & Quantitativo & 6 \\
\hline Rev. Latino-Am. Enfermagem ${ }^{(21)}$ & 2013 & Brasil & Quanti-Qualitativo & 6 \\
\hline Rev. Esc. Enferm. USP(22) & 2012 & Brasil & Rev. Integrativa & 5 \\
\hline Rev. Gaúcha Enferm. ${ }^{(23)}$ & 2013 & Brasil & Quantitativo & 6 \\
\hline Esc. Anna Nery ${ }^{(24)}$ & 2014 & Brasil & Qualitativo & 6 \\
\hline Enfermería Global ${ }^{(25)}$ & 2014 & Brasil & Quantitativo & 6 \\
\hline Cienc Cuid Saude ${ }^{(26)}$ & 2011 & Brasil & Documental & 5 \\
\hline Rev. enferm. UERJ. ${ }^{(27)}$ & 2013 & Brasil & Atualidade & 7 \\
\hline Rev. Esc. Enferm. USP(28) & 2012 & Brasil & Quantitativo & 6 \\
\hline Colomb Med $^{(29)}$ & 2011 & Brasil & Atualização & 7 \\
\hline Acta paul. enferm. ${ }^{(30)}$ & 2011 & Brasil & Qualitativo & 6 \\
\hline Gest. Prod ${ }^{(31)}$ & 2012 & Brasil & Qualitativo & 6 \\
\hline Salud UIS ${ }^{(32)}$ & 2013 & Colômbia & Qualitativo & 6 \\
\hline Rev. Latino-Am. Enfermagem ${ }^{(33)}$ & 2015 & Chile & Quantitativo & 6 \\
\hline Enferm Nefrol ${ }^{(34)}$ & 2012 & Chile & Revisão & 5 \\
\hline Gac. Sanit.(35) & 2012 & Espanha & Experiência & 6 \\
\hline J. Adv. Nurs. ${ }^{(36)}$ & 2013 & Áustria & Quantitativo & 6 \\
\hline J Nurs Scholarsh..$^{(37)}$ & 2014 & Coréia do Sul & Quantitativo & 6 \\
\hline J. Adv. Nurs. ${ }^{(38)}$ & 2013 & Austrália & Qualitativo & 6 \\
\hline Int Nurs Rev..$^{(39)}$ & 2015 & Tailândia & Quantitativo & 6 \\
\hline Nurs Outlook. ${ }^{(40)}$ & 2012 & Espanha & Quantitativo & 6 \\
\hline
\end{tabular}




\begin{tabular}{|c|c|c|c|c|}
\hline J. Adv. Nurs. ${ }^{(41)}$ & 2011 & Dinamarca & Quantitativo & 6 \\
\hline J Nurs Manag. ${ }^{(42)}$ & 2011 & EUA & Quantitativo & 6 \\
\hline J Nurs Manag. ${ }^{(43)}$ & 2015 & África & Quantitativo & 6 \\
\hline J Clin Nurs. ${ }^{(44)}$ & 2013 & Espanha & Retrospectivo & 6 \\
\hline Arch Psychiatr Nurs. ${ }^{(45)}$ & 2015 & China & Quantitativa & 6 \\
\hline Health Policy ${ }^{(46)}$ & 2010 & Canada & Documental & 6 \\
\hline Int J Nurs Stud. ${ }^{(47)}$ & 2014 & Alemanha & Quantitativo & 6 \\
\hline Int J Nurs Pract. ${ }^{(48)}$ & 2015 & Jordânia & Quantitativo & 6 \\
\hline J Clin Nurs. ${ }^{(49)}$ & 2012 & África & Quantitativo & 6 \\
\hline J Psychiatr Ment Health Nurs. ${ }^{(50)}$ & 2015 & Irlanda & Quantitativo & 6 \\
\hline J Adv Nurs. ${ }^{(51)}$ & 2013 & Noruega & Quantitativo & 6 \\
\hline J Nurs Scholarsh. ${ }^{(52)}$ & 2012 & Austrália & Quantitativo & 6 \\
\hline Int J Nurs Stud. ${ }^{(53)}$ & 2013 & EUA & Quantitativo & 6 \\
\hline Nurs Econ. ${ }^{(54)}$ & 2011 & EUA & Quantitativo & 6 \\
\hline Rev. Esc. Enferm. USP(55) & 2015 & Brasil & Quanti-qualitativo & 6 \\
\hline Saúde Debate $e^{(56)}$ & 2015 & Brasil & Qualitativo & 6 \\
\hline Texto Contexto Enferm. ${ }^{(57)}$ & 2015 & Brasil & Quanti-qualitativo & 6 \\
\hline
\end{tabular}

Com relação ao desfecho dos artigos, estes foram analisados, organizados e sintetizados para organização, interpretação e apresentação dos resultados, definindo-se as seguintes categorias: 1 . Conceitos e identificação de cargas de trabalho; 2. Condições de trabalho que predispõem as cargas de trabalho; 3. Influências das cargas de trabalho na saúde do trabalhador.

\section{- DISCUSSÃO}

\section{CATEGORIA 1 - Conceitos e identificação de cargas de trabalho}

A expressão "carga de trabalho" está relacionada às condições de trabalho(11) e fatores organizacionais ${ }^{(26)}$ a que os trabalhadores estão expostos. Assim, as cargas de trabalho estão presentes nos ambiente e processo de trabalho dos profissionais de enfermagem, podendo ser identificadas como cargas de materialidade interna, caracterizadas pelas cargas fisiológicas e psíquicas, pois se manifestam por meio de um distúrbio ou doença, e de materialidade externa, cargas físicas, químicas, biológicas e mecânicas, uma vez que são observadas no ambiente de trabalho ${ }^{(29)}$.

Em um hospital universitário, no ano de 2009, os trabalhadores de enfermagem referiram 160 cargas de trabalho, identificadas $37,5 \%$ como cargas fisiológicas, 36,2\% psíquicas, $14,4 \%$ biológicas, $7,5 \%$ mecânicas, 2,5\% físicas e 1,9\% químicas ${ }^{(8)}$. Em outro hospital universitário também em 2009, ocorreram 128 casos de adoecimento relacionado ao trabalho, e as cargas com maior destaque foram as fisiológicas e mecânicas com 33,06\% cada $^{(23)}$.

Em estudo realizado com sete hospitais públicos e universitários do Brasil também identificou que a exposição às cargas de trabalho está relacionada aos problemas de saúde dos trabalhadores de enfermagem, evidenciando a prevalência das cargas fisiológicas (36,8\%), biológicas (27,2\%), mecânicas $(25,9 \%)$ e psíquicas $(18,9 \%)^{(21)}$.

As cargas de trabalho apresentam fatores predominantes, nas cargas físicas estes fatores podem ser identificados por falta de espaço físico; salas apertadas, que dificultam a circulação; ausência de equipamentos, com manutenção precária ou ultrapassada; postura em pé ou desconforto em membros inferiores $^{(14)}$.

As cargas psíquicas são apresentadas por meio da identificação de desgastes relacionados à agressão psíquica, trabalho monótono e repetitivo, atenção constante, trabalho feminino, ritmo acelerado de trabalho, falta de comunicação, entre outras ${ }^{(16)}$. Outros fatores determinantes de carga psíquica estão relacionados aos relacionamentos interpessoais, responsabilidade decorrente do exercício 
profissional e risco de contaminação(14). Assim como, os trabalhadores que exercem atividades por turnos e acumulam funções apresentam índice médio de carga psíquica ${ }^{(13)}$.

\section{CATEGORIA 2 - Condições de trabalho que predispõem às cargas de trabalho}

A exposição à carga psíquica no trabalho da enfermagem pode estar relacionada com a tensão existente no ambiente de trabalho ${ }^{(10,22,47)}$ e a intensificação do trabalho ${ }^{(36)}$ por meio de exigências cognitivas e emocionais.

O ritmo de trabalho dos trabalhadores de enfermagem também é destaque nas produções científicas ${ }^{(19,31,33,37)}$, assim como a carga horária excessiva de trabalho exercida pelos trabalhadores ${ }^{(15,17,19,27-28,32,34,39,42-43)}$, dimensionamento inadequado ${ }^{(55-56)}$, dificuldade nas relações interpessoais ${ }^{(55)}$, e estes são considerados fatores determinantes da carga psíquica.

As cargas fisiológicas podem estar relacionadas ao esforço físico realizado pelo trabalhador durante o desenvolvimento do trabalho, evidenciando-se a realização das atividades com posturas em pé, percorrendo longas distâncias dentro da instituição, além de fatores ergonômicos, como manipulação e levantamento de peso e posturas inadequadas ${ }^{(12,18,20,31)}$, assim como a realização do trabalho em turnos ${ }^{(35)}$, e o desenvolvimento do trabalho no turno noturno ${ }^{(9,15,25,30,38,40,45-46,51)}$.

A manipulação do paciente ${ }^{(48)}$ e realização de banho de leito ${ }^{(57)}$ também causam problemas na saúde do trabalhador, sendo consideradas uma carga fisiológica. O uso de elevadores para elevação de paciente é uma solução para evitar a carga fisiológica, e as exigências físicas do trabalho ${ }^{(36,41)}$.

Os acidentes com perfurocortantes, durante a manipulação do material e agressões físicas ${ }^{(44,52,54)}$ são identificados como cargas mecânicas, já a exposição a material biológico, em casos de acidentes de trabalho, evidencia a carga biológica ${ }^{(24)}$. A carga química é descrita durante a exposição dos profissionais de enfermagem a agentes químicos e poluição ambiental ${ }^{(22,34)}$.

As cargas físicas estão relacionadas à falta de material ${ }^{(12,50)}$ e equipamentos ${ }^{(43,45,49)}$, barulho de máquinas ${ }^{(12)}$, manutenção e utilização de $\operatorname{mobiliários}^{(31)}$ e infraestrutura inadequada ${ }^{(49-50)}$.

\section{CATEGORIA 3 - Influências das cargas de trabalho na saúde do trabalhador}

As principais doenças, causas e notificações de afastamentos do trabalho são decorrentes do sistema osteoconjuntivo e tecido muscular ${ }^{(8)}$, osteoarticular e por traumas ${ }^{(23)}$, doenças do trato digestivo, oftalmológico e psíquico ${ }^{(25)}$, distúrbios osteomusculares ${ }^{(57)}$ de região lombar, pescoço, ombro e parte alta do dorso e extremidades superiores distais e membros inferiores ${ }^{(18,20,22,25)}$, dor musculoesquelética ${ }^{(53)}$ e dor nas $\operatorname{costas}^{(48)}$.

As cargas de trabalho apresentam um impacto negativo para a capacidade fisiológica dos trabalhadores ${ }^{(40)}$, evidenciando-se maior índice de massa corpórea e escores elevados de depressão nos trabalhadores de enfermagem ${ }^{(51)}$. Entre as reações fisiológicas do estresse presentes nos trabalhadores de enfermagem, são identificadas as dores lombares, fadiga e sensação de exaustão, rigidez no pescoço e acidez estomacal, entretanto o estudo não comprova a correlação entre carga de trabalho e as reações citadas ${ }^{(17)}$.

Outras consequências estão voltadas ao desgaste emocional ${ }^{(11,16,23,27,33,36,39,49)}$, associado à ocorrência de acidentes e problemas de saúde ${ }^{(11)}$, preocupação, perda de sono, ansiedade, medo, descontrole emocional, problemas no relacionamento com a família ${ }^{(24)}$, desgaste físico e emocional ${ }^{(27,29,56)}$. A falha de memória, dificuldade de concentração, medo de cometer erros ${ }^{(13)}$, e perturbações do sono ${ }^{(13,37-38)}$ também são influências das cargas de trabalho na saúde do trabalhador.

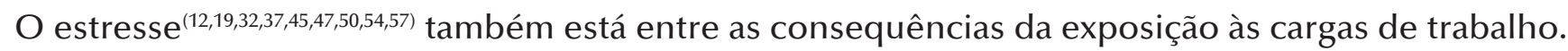
Outros fatores estão relacionados com o trabalho noturno, que perturba o equilíbrio oxidanteantioxidante e/ou gera estresse oxidativo ${ }^{(9)}$ nos trabalhadores, assim como o sofrimento ${ }^{(10,14,15,52)}$, síndrome de Burnout ${ }^{(34,37,40,44,50)}$ entre os profissionais de enfermagem e o aumento do risco de acidentes ${ }^{(21)}$ de trabalho. A implantação de um novo sistema de turnos de trabalho, visando uma melhor distribuição 
de carga horária, possibilitaria uma redução no número de ausências no trabalho ${ }^{(35)}$.

Existe prevalência das cargas fisiológicas e psíquicas como aquelas que mais afastam os trabalhadores nas instituições ${ }^{(8)}$. Estas cargas apresentam como fatores que favorecem o absenteísmo as longas jornadas de trabalho, condições insatisfatórias e falta de equipamentos ${ }^{(43)}$, assim como a sobrecarga de trabalho ${ }^{(46)}$, o maior esforço físico dos profissionais de enfermagem ${ }^{(41)}$ e o trabalho noturno ${ }^{(25,46)}$.

A exposição dos trabalhadores de enfermagem às cargas de trabalho apresenta como consequência os afastamentos $^{(23-24,56)}$ e absenteísmo no trabalho ${ }^{(8,25-26,41,43,46,55)}$. Também acarretam prejuízos na qualidade de $\operatorname{vida}^{(28,30,34)}$ e comprometimento na qualidade do cuidado e na segurança dos pacientes ${ }^{(30,39,57)}$.

\section{CONSIDERAÇÕES FINAIS}

O estudo evidenciou que o processo e ambiente de trabalho da equipe de enfermagem são permeados por condições que predispõem às cargas de trabalho e que estas cargas causam influências na saúde do trabalhador.

Compreendendo o objetivo deste estudo, destacam-se, como influências das cargas de trabalho na saúde do trabalhador, as doenças e acidentes relacionados ao trabalho, os desgastes físicos, psíquicos e o absenteísmo.

Neste ínterim, conclui-se que as cargas de trabalho causam prejuízos à saúde dos trabalhadores de enfermagem, à instituição e à assistência segura ao paciente. Assim, são necessárias ações organizacionais, de prevenção de acidentes e doenças relacionados ao trabalho, visando à redução das cargas e à promoção da saúde do trabalhador de enfermagem.

\section{REFERÊNCIAS}

1. Silva GT, Cunha CRT, da Costa ALRC, Maruyama SAT. Experiência de adoecimento e licença médica: o caso de uma técnica de enfermagem. Reme, Rev. Min. Enferm. [Internet] 2013;17(1) [acesso em 15 nov 2016]. Disponível: http://www.reme.org.br/artigo/detalhes/591.

2. Rocha LP, Cezar-Vaz MR, de Almeida MCV, Borges AM, da Silva MS, Sena-Castanheira J. Workloads and occupational accidents in a rural environment. Texto Contexto Enferm. [Internet] 2015;24(2) [acesso em 15 nov 2016]. Disponível: http://dx.doi.org/10.1590/0104-07072015000480014.

3. Felli VEA. Condições de trabalho de enfermagem e adoecimento: motivos para a redução da jornada de trabalho para 30 horas. Enferm. foco. [Internet] 2012;3(4) [acesso em 15 nov 2016]. Disponível: http://revista.portalcofen. gov.br/index.php/enfermagem/article/view/379.

4. Prudente JAB, Salum NC, Gelbcke FL, Lorenzetti J, Schier J. O desgaste de trabalhadores de enfermagem no cuidado a pacientes onco-hematológicos hospitalizados. Cogitare Enferm. [Internet] 2015;20(1) [acesso em 15 nov 2016]. Disponível: http://dx.doi.org/10.5380/ce.v20i1.38299.

5. Karino ME, Felli VEA, Sarquis LMM, Santana LL, Silva SR, Teixeira RC. Cargas de trabalho e desgastes dos trabalhadores de enfermagem de um hospital-escola. Cienc Cuid Saude. [Internet] 2015;14(2) [acesso em 15 nov 2016]. Disponível: http://dx.doi.org/10.4025/cienccuidsaude.v14i2.21603.

6. Whittemore R, Knafl K. The integrative review: updated methodology. J. Adv. Nurs. [Internet] 2005;52(5) [acesso em 15 nov 2016]. Disponível: http://dx.doi.org/10.1111/j.1365-2648.2005.03621.x.

7. Melnyk BM, Fineout-Overholt E. Making the case for evidence-based practice.In: Melnyk BM, FineoutOverholt E. Evidence-based practice in nursing \& healthcare. A guide to best practice. Philadelphia: Lippincot Williams \& Wilkins. 2005; 3-24.

8. Mininel VA, Felli VEA, da Silva EJ, Torri Z, Abreu AP, Branco MTA. Cargas de trabalho, processos de desgaste e absenteísmo-doença em enfermagem. Rev. Latino-Am. Enfermagem. [Internet] 2013;21(6) [acesso em 06 nov 2016]. Disponível: http://dx.doi.org/10.1590/0104-1169.2992.2366. 
9. Gromadzin'ska J, Peplonska WS, Reszka E, Wasowicz W, Bukowska A, et al. Relationship between intensity of night shift work and antioxidant status in blood of nurses. Int. Arch. Occup. Environ. Health. [Internet] $2013 ; 86$ [acesso em 20 nov 2016]. Disponível: http://dx.doi.org/10.1007/s00420-012-0828-7.

10. Tzeng DS, Chung WC, Yang CY. The Effect of Job Strain on Psychological Morbidity and Quality of Life in Military Hospital Nurses in Taiwan: A Follow-up Study. Ind. Health. [Internet] 2013;51(4) [acesso em 15 nov 2016]. Disponível: http://doi.org/10.2486/indhealth.2012-0013.

11. Schmoeller R, Trindade LL, Neis MB, Gelbcke FL, de Pires DEP. Cargas de trabalho e condições de trabalho da enfermagem: revisão integrativa. Rev. gauch. enferm. [Internet] 2011;32(2) [acesso em 05 nov 2016]. Disponível: http://dx.doi.org/10.1590/S1983-14472011000200022.

12. Beserra FM, Alves e Souza AM, Moreira DA, Alves MDS, D'Alencar BP. Significado do trabalho dos profissionais de enfermagem no hospital geral. Av. enferm. [Internet] 2010;28(2) [acesso em 10 nov 2016]. Disponível: http:// www.scielo.org.co/pdf/aven/v28n2/v28n2a03.pdf.

13. Ferreira MM, Ferreira C. Carga mental e carga psíquica em profissionais de enfermagem. Revista Portuguesa de Enfermagem de Saúde Mental. [Internet] 2014;(n.esp 1). [acesso em 06 nov 2016]. Disponível: http://www. scielo.mec.pt/pdf/rpesm/nspe1/nspe1a08.pdf.

14. da Silva NR, Fatores determinantes da carga de trabalho em uma unidade básica de saúde. Ciênc. saúde coletiva. [Internet] 2011;16(8) [acesso em 06 nov 2016]. Disponível: http://dx.doi.org/10.1590/S1413-81232011000900006.

15. Campos RO, Baccari IP. A intersubjetividade no cuidado à Saúde Mental: narrativas de técnicos e auxiliares de enfermagem de um Centro de Atenção Psicossocial. Ciênc. saúde coletiva. [Internet] 2011;16(4) [acesso em 05 nov 2016]. Disponível: http://dx.doi.org/10.1590/S1413-81232011000400004.

16. Mininel VA, Baptista PCP, Felli VEA. Cargas psíquicas e processos de desgaste em trabalhadores de enfermagem de hospitais universitários brasileiros. Rev. Latino-Am. Enfermagem. [Internet] 2011;19(2) [acesso em 06 nov 2016]. Disponível: http://www.scielo.br/pdf/rlae/v19n2/pt_16.

17. Dalri RCMB, da Silva LA, Mendes AMOC, Robazzi MLCC. Nurses' workload and its relation with physiological stress reactions. Rev. Latino-Am. Enfermagem. [Internet] 2014;22(6) [acesso em 06 nov 2016]. Disponível: http:// dx.doi.org/10.1590/0104-1169.3292.2503.

18. Ribeiro NF, Fernandes RCP, Solla DJF, Santos Junior AC, de Sena Junior AS. Prevalência de distúrbios osteomusculares relacionados ao trabalho em profissionais de enfermagem. Rev. bras. epidemiol. [Internet] 2012; 15(2) [acesso em 10 nov 2016]. Disponível: http://dx.doi.org/10.1590/S1415-790X2012000200020.

19. da Silva JLL, dos Santos RSFB, Costa FS, Taveira RPC, Teixeira LR. Estressores na atividade gerencial do enfermeiro: implicações para saúde. Av. enferm. [Internet] 2013;31(2) [acesso em 06 nov 2016]. Disponível: http:// www.scielo.org.co/pdf/aven/v31n2/v31n2a15.pdf.

20. Fonseca NR, Fernandes RCP. Factors Related to Musculoskeletal Disorders in Nursing Workers. Rev. LatinoAm. Enfermagem. [Internet] 2010;18(6) [acesso em 10 nov 2016]. Disponível: http://dx.doi.org/10.1590/S010411692010000600006.

21. da Silva SM, Baptista PCP, Felli VEA, Martins AC, Sarquis LMM, Mininel VA. Intervention strategies for the health of university hospital nursing staff in Brazil. Rev. Latino-Am. Enfermagem. [Internet] 2013;21(1) [acesso em 05 nov 2016]. Disponível: http://dx.doi.org/10.1590/S0104-11692013000100003.

22. Ribeiro RP, Martins JT, Marziale MHP, Robazzi MLCC. O adoecer pelo trabalho na enfermagem: uma revisão integrativa. Rev. esc. enferm. USP. [Internet] 2012;46(2) [acesso em 06 nov 2016]. Disponível: http://dx.doi. org/10.1590/S0080-62342012000200031.

23. Santana LL, Miranda FMDA, Karino ME, Baptista PCP, Felli VEA, Sarquis LMM. Cargas e desgastes de trabalho vivenciados entre trabalhadores de saúde em um hospital de ensino. Rev. gaúch. enferm. [Internet] 2013;34(1) [acesso em 06 nov 2016]. Disponível: http://dx.doi.org/10.1590/S1983-14472013000100008.

24. Marziale MHP, dos Santos HEC, Cenzi CM, Rocha FLR, Trovó MEM. Consequências da exposição ocupacional a material biológico entre trabalhadores de um hospital universitário. Esc. Anna Nery. [Internet] 2014;18(1) [acesso em 25 nov 2016]. Disponível: http://dx.doi.org/10.5935/1414-8145.20140002. 
25. Trindade LL, Grisa CC, Ostrovki V P, Adamy EK, Ferraz L, Amestoy SC, Bordignon M. Absentismo en el equipo de enfermería en el ambiente hospitalario. Enfermería Global. [Internet] 2014;13(4) [acesso em 20 nov 2016$].$ Disponível: http://dx.doi.org/10.6018/eglobal.13.4.181541.

26. Umann J, Guido LA, Freitas EO. Produção de conhecimento sobre saúde e doença na equipe de enfermagem na assistência hospitalar. Cienc Cuid Saude. [Internet] 2011;10(1) [acesso em 05 nov 2016]. Disponível: http:// dx.doi.org/10.4025/cienccuidsaude.v10i1.10931.

27. dos Santos TA, da Silva OS, de Melo CMM, Costa HOG. Significado da regulamentação da jornada de trabalho em enfermagem. Rev. enferm. UERJ. [Internet] 2013;21(2) [acesso em 06 nov 2016]. Disponível: http://www.facenf. uerj.br/v21n2/v21n2a21.pdf.

28. Fernandes JS, Miranzi SSC, Iwamoto HH, Tavares DMS, dos Santos CB. A relação dos aspectos profissionais na qualidade de vida dos enfermeiros das equipes saúde da família. Rev Esc Enferm USP. [Internet] 2012;46(2) [acesso em 06 nov 2016]. Disponível: http://dx.doi.org/10.1590/S0080-62342012000200019.

29. Kirchhof ALC, Lacerda MR, Sarquis LMM, Magnago TSB, Gomes IM. Compreendendo cargas de trabalho na pesquisa em saúde ocupacional na enfermagem. Colomb Med. [Internet] 2011;4(2 Suppl. 1) [acesso em 05 nov 2016]. Disponível: http://www.bioline.org.br/pdf?rc11047.

30. de Oliveira EM, Spiri WC. Dimensão pessoal do processo de trabalho para enfermeiras de Unidades de Terapia Intensiva. Acta paul. enferm. [Internet] 2011;24(4) [acesso em 25 nov 2016]. Disponível: http://dx.doi. org/10.1590/S0103-21002011000400016.

31. Mendes DP, de Oliveira MM, de Matos VG, Mazoni MB, Moraes GFS. Do prescrito ao real: a gestão individual e coletiva dos trabalhadores de enfermagem frente ao risco de acidente de trabalho. Gest. Prod. [Internet] 2012;19(4) [acesso em 06 nov 2016]. Disponível: http://dx.doi.org/10.1590/S0104-530X2012000400016.

32. Giraldo YG, Gómez BL, David LA, Henao FG, Silva E. Estrés laboral en auxiliares de enfermeira. Salud UIS. 2013;45(1) [acesso em 15 nov 2016]. Disponível: http://www.scielo.org.co/pdf/suis/v45n1/v45n1a04.pdf.

33. Ceballos-Vásquez P, Rolo-González G, Hernández-Fernaud E, Díaz-Cabrera D, Paravic-Klijn T, Burgos-Moreno M. Psychosocial factors and mental work load: a reality perceived by nurses in intensive care units. Rev. LatinoAm. Enfermagem. [Internet] 2015;23(2) [acesso em 05 nov 2016]. Disponível: http://dx.doi.org/10.1590/01041169.0044.2557.

34. Araya SB, Mantuliz MCA, Parada VV. Relación entre carga laboral y burnout en enfermeiras de unidades de diálisis. Enferm Nefrol. [Internet] 2012;15(1) [acesso em 15 nov 2016]. Disponível: http://scielo.isciii.es/scielo. php?script=sci_arttext\&pid=S2254-28842012001100007.

35. Gutiérrez JJB, González AR, Ábalos MÁG, Herrera AA, de Rivera JCMA, González AA. Efecto de la implantación de turnos de enfermería «a demanda» sobre las horas de absentismo. Gac. Sanit. [Internet] 2012;26(5) [acesso em 20 nov 2016]. Disponível: http://scielo.isciii.es/pdf/gs/v26n5/nota_campo.pdf.

36. Kubicek B, Korunka C, Ulferts H. Acceleration in the care of older adults: new demands as predictors of employee burnout and engagement. J. Adv. Nurs. [Internet] 2013;69(7) [acesso em 15 nov 2016]. Disponível: http://dx.doi.org/10.1111/jan.12011.

37. Cho SH, Park M, Jeon SH, Chang HE, Hong HJ. Average hospital length of stay, nurses' work demands, and their health and job outcomes. J Nurs Scholarsh. [Internet] 2014;46(3) [acesso em 15 nov 2016]. Disponível: http:// dx.doi.org/10.1111/jnu.12066.

38. Powel I. Can you see me? Experiences of nurses working night shift in Australian regional hospitals: a qualitative case study. J. Adv. Nurs. [Internet] 2013;69(10) [acesso em 20 nov 2016]. Disponível: http://dx.doi. org/10.1111/jan.12079.

39. Kunaviktikul W, Wichaikhum O, Nantsupawat A, Nantsupawat R, Chontawan R, Klunklin A, et al. Nurses' extended work hours: Patient, nurse and organizational outcomes. Int Nurs Rev. [Internet] 2015;62(3) [acesso em 15 nov 2016]. Disponível: http://dx.doi.org/10.1111/inr.12195.

40. García-Izquierdo M, Ríos-Rísquez MI. The relationship between psychosocial job stress and burnout in emergency departments: an exploratory study. Nurs Outlook. [Internet] 2012;60(5) [acesso em 20 nov 2016$].$ Disponível: http://dx.doi.org/10.1016/j.outlook.2012.02.002. 
41. Jensen JN, Holten AL, Karpatshof B, Albertsen K. Does collective efficacy moderate the associations between physical work load and intention to leave or sickness absence? J. Adv. Nurs. [Internet] 2011;67(11) [acesso em 20 nov 2016]. Disponível: http://dx.doi.org/10.1111/j.1365-2648.2011.05674.x.

42. Chen J, Davis LS, Davis KG, Pan W, Daraiseh NM. Physiological and behavioural response patterns at work among hospital nurses. J Nurs Manag. [Internet] 2011;19(1) [acesso em 15 nov 2016]. Disponível: http://dx.doi. org/10.1111/j.1365-2834.2010.01210.x.

43. Mundaly P, Nkosi ZZ. Factors influencing nurse absenteeism in a general hospital in Durban, South Africa. J Nurs Manag. [Internet] 2015;23(5) [acesso em 15 nov 2016]. Disponível: http://dx.doi.org/10.1111/jonm.12189.

44. Gascon S, Leiter MP, Andrés E, Santed MA, Pereira JP, Cunha MJ, et al. The role of aggressions suffered by healthcare workers as predictors of burnout. J Clin Nurs. [Internet] 2013;22(21-22) [acesso em 22 nov 2016]. Disponível: http://dx.doi.org/10.1111/j.1365-2702.2012.04255.x.

45. Lu DM, Sun N, Hong S, Fan YY, Kong FY, Li QJ. Occupational Stress and Coping Strategies Among Emergency Department Nurses of China. Arch Psychiatr Nurs. [Internet] 2015;29(4) [acesso em 20 nov 2016]. Disponível: http://dx.doi.org/10.1016/j.apnu.2014.11.006.

46. Rajbhandary S, Basu K. Working conditions of nurses and absenteeism: is there a relationship? an empirical analysis using National Survey of the Work and Health of Nurses. Health policy. [Internet] 2010;97(2-3) [acesso em 20 nov 2016]. Disponível: http://dx.doi.org/10.1016/j.healthpol.2010.04.010.

47. Schmidt KH, Diestel S. Are emotional labour strategies by nurses associated with psychological costs? A cross-sectional survey. Int J Nurs Stud. [Internet] 2014;51(11) [acesso em 15 nov 2016]. Disponível: http://dx.doi. org/10.1016/j.ijnurstu.2014.03.003.

48. Shawashi TO, Subih MM, Al Hadid LAR, Abu Adas M. Occupational-related back pain among Jordanian nurses: A descriptive study. Int J Nurs Pract. [Internet] 2015;21(Suppl 2) [acesso em 20 nov 2016]. Disponível: http://dx.doi.org/10.1111/ijn.12168.

49. van der Doef $M$, Mbazzi B, Verhoeven C. Job conditions, job satisfaction, somatic complaints and burnout among East African nurses. J Clin Nurs. [Internet] 2012;21(11-12) [acesso em 20 nov 2016]. Disponível: http:// dx.doi.org/10.1111/j.1365-2702.2011.03995.x.

50. Mctiernan K, Mcdonald N. Occupational stressors, burnout and coping strategies between hospital and community psychiatric nurses in a Dublin region. J Psychiatr Ment Health Nurs. [Internet] 2015;22(3) [acesso em 20 nov 2016]. Disponível: http://dx.doi.org/10.1111/jpm.12170.

51. Saksvik-Lehouillier I, Bjorvan B, Hetland H, Sandal GM, Moen BE, Mageroy N, et al. Individual, situational and lifestyle factors related to shift work tolerance among nurses who are new to and experienced in night work. J Adv Nurs. [Internet] 2013;69(5) [acesso em 20 nov 2016]. Disponível: http://dx.doi.org/10.1111/j.13652648.2012.06105.x.

52. Demir D, Rodwell J. Psychosocial Antecedents and Consequences of Workplace Aggression for Hospital Nurses. J Nurs Scholarsh. [Internet] 2012;44(4) [acesso em 22 nov 2016]. Disponível: http://dx.doi.org/10.1111/ j.1547-5069.2012.01472.x.

53. Lee SJ, Faucett J, Gillen M, Krause N. Musculoskeletal pain among critical-care nurses by availability and use of patient lifting equipment: An analysis of cross-sectional survey data. Int J Nurs Stud. [Internet] 2013;50(12) [acesso em 22 nov 2016]. Disponível: http://dx.doi.org/10.1016/j.ijnurstu.2013.03.010.

54. Gates DM, Gillespie GL, Succop P. Violence Against Nurses and its Impact on Stress and Productivity. Nurs Econ. [Internet] 2011;29(2) [acesso em 20 nov 2016]. Disponível: http://www.medscape.com/viewarticle/746092.

55. Kurgant P, Passos AR, de Oliveira JML, Pereira IM, Costa TF. Absenteísmo do pessoal de enfermagem: decisões e ações de enfermeiros gerentes. Rev. esc. enferm. USP. [Internet] 2015;49(n.esp2) [acesso em 20 nov 2016]. Disponível: http://dx.doi.org/10.1590/S0080-623420150000800005.

56. de Souza SJP, Larocca LM, Chaves MMN, Alessi SM. A realidade objetiva das doenças e agravos não transmissíveis na enfermagem. Saúde debate. [Internet] 2015;39(106) [acesso em 20 nov 2016]. Disponível: http:// dx.doi.org/10.1590/0103-110420151060003007. 
57. Möller G, de Magalhães AMM. Banho no leito: carga de trabalho da equipe de enfermagem e segurança do paciente. Texto Contexto Enferm. [Internet] 2015;24(4) [acesso em 20 nov. 2016]. Disponível: http://dx.doi. org/10.1590/0104-0707201500003110014. 